\title{
Molecular diagnosis in type I epithelial ovarian cancer
}

\author{
Paweł Sadłecki, Małgorzata Walentowicz-Sadłecka, Marek Grabiec \\ Department of Obstetrics and Gynecology, L. Rydygier Collegium Medicum in Bydgoszcz, \\ Nicolaus Copernicus University, Bydgoszcz, Poland
}

\begin{abstract}
The term epithelial ovarian cancer refers to a heterogeneous group of tumors, including serous, mucinous, endometrioid and clear cell carcinomas, each characterized by specific molecular background and clinical outcome. A growing body of evidence suggests that molecular pathogenesis of ovarian cancer involves two general pathways. The first pathway results in transformation of normal ovarian tissue to borderline tumors, which may further progress to low-grade serous, mucinous, endometrioid and clear cell carcinomas. Type I tumors often harbor somatic mutations in protein kinase genes, as well as in genes for other signaling molecules. Both BRAF and KRAS mutations lead to a constitutive activation of their downstream target, mitogen-activated protein kinase. Identification of molecular profile may be crucial for the diagnosis of ovarian tumors, choice of adjuvant targeted therapy and management of recurrence disease. Point mutations in cancer cells can be detected with many various methods. Application of direct sequencing as a routine method for cytological diagnosis used in a hospital setting requires expensive equipment and implementation of complicated procedures. Another factor limiting application of this method in everyday clinical practice are long analytical times. This stimulated search for a simple, rapid, specific and sensitive methodology to detect point mutations. Recently, some new molecular assays for the detection of BRAF, KRAS and NRAS mutations have become available. These are fully-automated molecular diagnostic systems for quantitative allele-specific RT-PCR-based analyses. Using this instrument, even pathologists from less experienced laboratories can easily integrate morphological findings with molecular data being crucial for further diagnostic and therapeutic decisions.
\end{abstract}

Key words: low grade ovarian cancer, type 1 ovarian cancer, borderline ovarian tumors, KRAS mutation, BRAF mutation, NRAS mutation

\section{INTRODUCTION}

Epithelial ovarian cancer (EOC) represents approximately $90 \%$ of all primary ovarian malignancies. The term EOC refers to a heterogeneous group of tumors, including serous, mucinous, endometrioid and clear cell carcinomas, each characterized by specific molecular background and clinical outcome. A growing body of evidence suggests that molecular pathogenesis of ovarian cancer involves two general pathways [1]. The first pathway results in transformation of normal ovarian tissue to borderline tumors, which may further progress to low-grade serous, mucinous, endometrioid and clear cell carcinomas. Type I tumors often harbor somatic mutations in protein kinase genes (KRAS, BRAF, $\mathrm{PI} 3 \mathrm{KCA}$ and ERRB2), as well as in genes for other signaling molecules (e.g. CTNNB1 and PTEN). Tumors from this group are characterized by slow proliferation, and approximately
$55 \%$ 5-year survival rate [2]. In contrast, type II ovarian tumors are high-grade, highly aggressive malignancies, spreading rapidly throughout the pelvis. This group includes high-grade serous carcinoma, malignant mixed mesodermal tumors and undifferentiated carcinomas. Many of these malignancies harbor mutations in TP53, a tumor suppressor gene, and have high proliferative index. It is estimated that $60 \%$ of sporadic ovarian malignancies and most tumors found in BRCA1 mutation carriers are high-grade serous carcinomas [3].

RAS/mitogen-activated protein kinase (MAPK) and phosphatidylinositol-4,5-bisphosphate 3-kinase (PI3K)/protein kinase $B(A K T)$ are two major intracellular signaling transduction pathways, activated due to: loss of phosphatase and tensin homolog (PTEN) function, genetic mutations in phosphatidylinositol-4,5-bisphosphate 3-kinase catalytic 
subunit a (PI3KCA), Kirsten rat sarcoma viral oncogene homolog (KRAS), neuroblastoma RAS viral oncogene homolog (NRAS) or proto-oncogene serine/threonine kinase B-Raf (BRAF), or induction of mutations in MET proto-oncogene, a member of the receptor tyrosine kinase family being involved in ovarian carcinogenesis [4, 5]. Both BRAF and KRAS mutations lead to a constitutive activation (phosphorylation) of their downstream target, mitogen-activated protein kinase (MAPK), also referred to as extracellular signal-regulated protein kinase (ERK) [6]. Presence of mutations in BRAF or KRAS genes co-exists with overexpression of activated ERK $1 / 2$ in ovarian serous tumors. Upregulation of ERK $1 / 2$ results in activation of downstream cellular targets, including a variety of cellular and nuclear proteins [7]. However, biological role of this pathway in the development of ovarian malignancies other than serous ovarian cancer is still not completely understood.

Identification of molecular profile may be crucial for the diagnosis of ovarian tumors, choice of adjuvant targeted therapy after primary cytoreductive treatment, or management of recurrence in patients with advanced type I epithelial ovarian neoplasms.

\section{LOW-GRADE SEROUS OVARIAN CANCER}

Low-grade serous ovarian cancer (LGSC) is characterized by young age at diagnosis, indolent course, and longer overall survival than in the case of high-grade serous ovarian cancers (HGSCs), even at more advanced stages [2]. LGSCs represent less than $10 \%$ of serous ovarian cancers and are less sensitive to conventional platinum-based chemotherapy than HGSCs [8]. They are characterized by relative nuclear uniformity, lack of nuclear pleomorphism, and mitotic activity no greater than 12 mitoses per 10 high-power fields. Under a microscope, LGSCs typically present as micropapillae surrounded by clear spaces; however, glandular, macropapillary and single cell patterns can also be seen (Fig. 1) [9]. LGSCs are believed to arise from preexisting cystadenomas or serous borderline tumors (SBTs) that eventually progress to an invasive carcinoma. While the majority of typical SBTs do not have a typical phenotype of invasive carcinoma, microinvasion is not uncommon. In some studies, up to $60 \%$ LGSCs were associated with SBTs [10]. Although HGSCs and LGSCs share some characteristics, they have also some distinct immunophenotypic features. Both tumor types express paired box gene 8 (PAX8) and WT1, as well as estrogen (ER) and progesterone receptors (PR) [9]. LGSCs are characterized by patch $p 16$ staining pattern, wild-type p53 staining pattern, and low Ki-67 proliferative index [11]. More than $60 \%$ of LGSCs harbor MAPK pathway-activating KRAS or BRAF mutations (occurring with a similar frequency of ca. $30 \%$ ); mutations in HER2 have been also reported [12]. Both Grisham and Wong reported that women

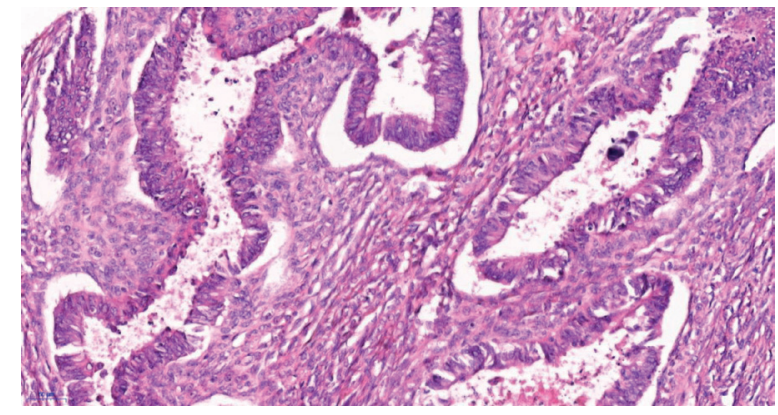

Figure 1. Microphotograph presenting H\&E stained specimens of low grade serous ovarian cancer, primary objective magnification $20 \times$

with mutations in KRAS and/or BRAF had better prognosis than those without. This phenomenon can be at least partially explained by the results of previous studies. SBTs from women with BRAF mutations were shown to over-express genes with cell growth inhibitory effects [13]. Furthermore, activating BRAF mutations were postulated to induce cellular senescence and to prevent progression of SBTs to LGSCs [14]. On the other hand, Singer reported that presence of KRAS mutation may be associated with more aggressive, recurrent phenotype of LGSC than the one observed in BRAF mutation carriers [15]. It should be emphasized that presence of KRAS mutation in a given tumor excludes existence of a concomitant BRAF mutation, and vice versa [15].

\section{ENDOMETRIOID OVARIAN ADENOCARCINOMA}

Endometrioid ovarian adenocarcinoma (EC) is usually associated with endometriosis or endometrioid adenofibroma/borderline tumor. It typically presents as a unilateral mass confined to the ovary. In most cases, EC is diagnosed based on the presence of back-to-back glands, rather than infiltrative invasion [9]. EC has several characteristic histologic features, such as squamous morules, mucinous differentiation, clear cell change, spindle-shaped morphology and secretory change, although the latter may sometimes raise a suspicion of clear cell carcinoma (Fig. 2) [9]. EC should be primarily differentiated from metastatic colonic adenocarcinoma; differential diagnosis can be based on immunohistochemistry, since EC is positive for cytokeratin 7 (CK7), PAX8, ER and PR, whereas colonic adenocarcinoma tests positively for cytokeratin 20 (CK20) and caudal-type homeobox 2 (CDX2) protein. However, it should be remembered that the expression of CDX2 may be also found in squamous morules forming EC [16]. Distinguishing high-grade EC from a high-grade serous carcinoma (HGSC) may be challenging, especially in the case of predominantly solid tumors. In such cases, immunostaining for WT1, p53 and p16 is an option; usually, HGSCs give strong and diffuse positive reaction with these markers, whereas ECs are negative for WT1, show patch 


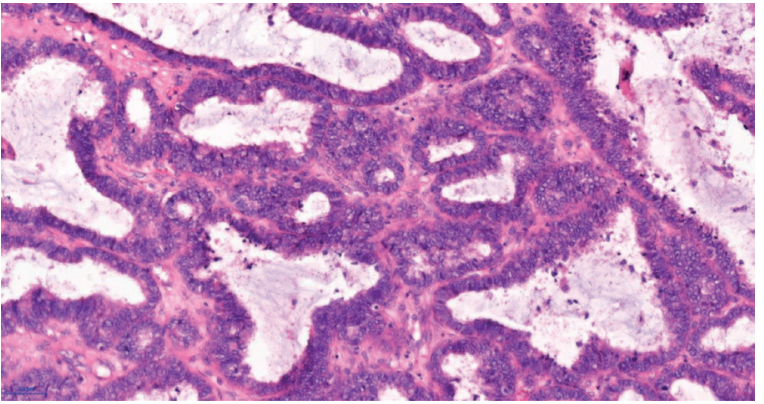

Figure 2. Microphotograph presenting $H \& E$ stained specimens of endometrioid ovarian adenocarcinoma, primary objective magnification 20x

p16 staining pattern and wild-type p53 staining pattern [17]. Approximately $10 \%$ of patients with ovarian cancers present with synchronous endometrial tumors [18], metastases from the ovary or endometrium, or synchronous primary ovarian or endometrial tumors. Soliman et al. found that patients with synchronous endometrial and ovarian primaries had distinct clinical characteristics, such as younger age, nulliparity, obesity and premenopausal status [18]. Overall survival in subjects with synchronous primaries is usually excellent (approximately 10 years), especially if both tumors had endometrioid histology. Ovarian ECs are typically associated with somatic mutations in catenin $\beta 1$ (CTNNB1) and PTEN genes [19]. The frequency of CTNNB1 abnormalities in ovarian and endometrial ECs is essentially the same, but the former less often show microsatellite instability and PTEN alterations. In rare cases, a low-grade EC may co-exist with an adjacent undifferentiated carcinoma; this type of malignancy is referred to as de-differentiated carcinoma, and can be often misdiagnosed as EC, FIGO grade 3. De-differentiated carcinoma was first described by Silva et al.; these authors demonstrated that presence of an undifferentiated carcinoma component of any size in ovarian or endometrial endometrioid carcinoma is associated with markedly worse prognosis [20]. In the ovaries, de-differentiated carcinomas may represent the far end of the HGSC spectrum or be associated with low-grade ECs. Undifferentiated carcinoma is composed of discohesive, monotonous cells resembling lymphoma. Identification of specific morphologic features of these tumors is vitally important, especially if they co-exist with low-grade ECs, since as already mentioned, such combination is associated with a significantly worse prognosis. Although undifferentiated carcinomas can express some neuroendocrine markers, proportion of these tumors with a positive result of immunostaining is less than $10 \%$. Diffuse pattern of immunostaining for neuroendocrine markers favors diagnosis of a high-grade neuroendocrine carcinoma [21]. EC has an excellent prognosis, especially when confined to the ovary. In adequately staged patients, an acceptable therapeutic strategy can be even a watchful waiting.

\section{MUCINOUS OVARIAN CANCER}

Mucinous ovarian cancer is a rare histological type, representing 2-4\% of all epithelial ovarian carcinomas [22]. Mucinous tumors have a multicystic structure with more than $90 \%$ of cells filled with conspicuous amounts of mucin (usually occupying $\geq 50 \%$ of the cytoplasm) [22]. Origin of mucinous tumors still raises some controversies. Many malignancies from this group that were previously classified as primary tumors, are nowadays considered misdiagnosed metastases from other locations, primarily from the gastrointestinal tract and endocervix (Fig. 3) [23]. Mucinous ovarian tumors are postulated to arise from benign lesions, which transform to mucinous borderline tumors and then, to the invasive forms. The majority of them are mucinous borderline tumors (MBOTs) or stage I mucinous carcinomas (MCs). Although prognosis is generally excellent, in rare cases with extra-ovarian spread, the outcomes and response to conventional chemotherapy may be poor. Aside from sharing many biomarkers with pancreatic and gastrointestinal adenocarcinomas, MCs have also similar morphology; therefore, differentiating between primary ovarian tumors and metastatic disease may sometimes pose a challenge [24]. Primary mucinous ovarian carcinomas differ from other epithelial ovarian cancers in both presentation and outcome [25]. A number of features shared by mucinous tumors, among them a dominance of RAS-activating changes, raises hopes for the development of targeted therapeutic strategies dedicated to women with advanced or recurrent disease [26]. The most common genetic defects found in mucinous tumors are abnormalities of mitogen activated protein kinase (MAPK) pathway, especially mutations of KRAS genes and amplification/overexpression of ERBB2 [26]. Evidence from previous studies suggests that prognosis in mucinous ovarian cancers without a known alteration of RAS-pathway may be unfavorable, likewise in low-grade serous/serous borderline ovarian tumors [27]. According to literature, more than $75 \%$ of mucinous ovarian tumors may harbor KRAS mutations; however, it should be remembered that authors of some of these reports did not differentiate

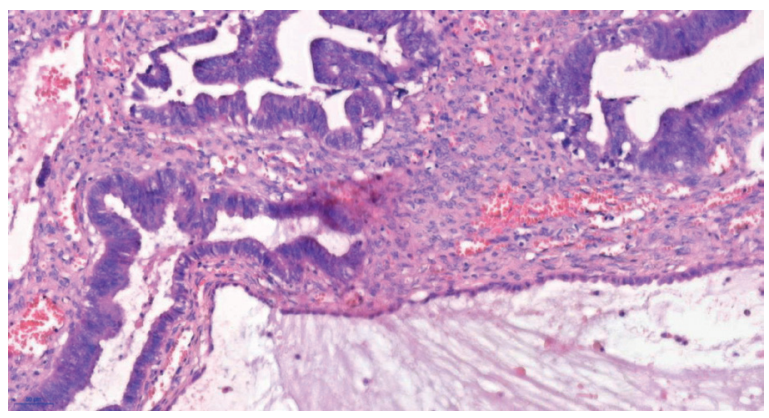

Figure 3. Microphotograph presenting H\&E stained specimens of mucinous ovarian cancer, primary objective magnification $20 \times$ 
between MBOTs and MCs, and/or did not exclude metastatic lesions from mutational status analysis [28]. The results of copy number analysis imply that development of mucinous tumors may be associated with the loss of heterozygosity of chromosomal regions $9 p, 17 p$ and $21 q$. Also other mutated genes, BRAF, TP53, PTEN, PI3KCA, and more recently also CDKN2A and RNF43, have been found in tumors from this group. However, the rarity of mucinous ovarian tumors markedly hinders the possibility of large-scale mutational frequency analyses [29].

\section{CLEAR CELL OVARIAN CANCER}

Clear cell ovarian cancer (CCC) represents $5-13 \%$ of all epithelial ovarian malignancies [2]. CCCs are usually diagnosed at a younger age than high-grade serous epithelial ovarian cancers. They usually present as a pelvic mass confined to the ovary, often co-existing with endometriosis (Fig. 4) [30]. CCCs have an aggressive clinical phenotype and rarely respond to standard platinum-based chemotherapy; due to such characteristics, prognosis in CCC is worse than in HGSC [31]. Recent evidence suggests that approximately $40 \%$ of these tumors test positively for PI3KCA mutations, which implies that their pathogenesis may involve some aberrations in telomere biology [32]. The majority of CCCs express wild-type $\mathrm{p} 53$, rarely harbor germline mutations in BRCA 1 and BRCA 2, and show low level of chromosomal instability [33]. Another characteristic feature of CCCs is high level of hypoxia inducible factor 1 alpha (HIF1a) [34]. The proportion of CCCs expressing HER2/neu is 2.5- to 10-fold higher than in the case of type I and II ovarian tumors, which points to a potential role for HER2/neu inhibitors in treatment of these malignancies. Published data documenting KRAS mutational status of CCCs are generally sparse and inconclusive [35]. Jones and Auner et al. detected KRAS mutations in $4.7 \%$ and $26 \%$ of CCCs, respectively [36, 37]. In contrast, Rechsteineret al. found no mutations of this gene in a large cohort of CCC patients [38]. According to Despierre et al., mutations of KRAS are present in $14 \%$ of CCCs, which is markedly lower proportion than in other subtypes

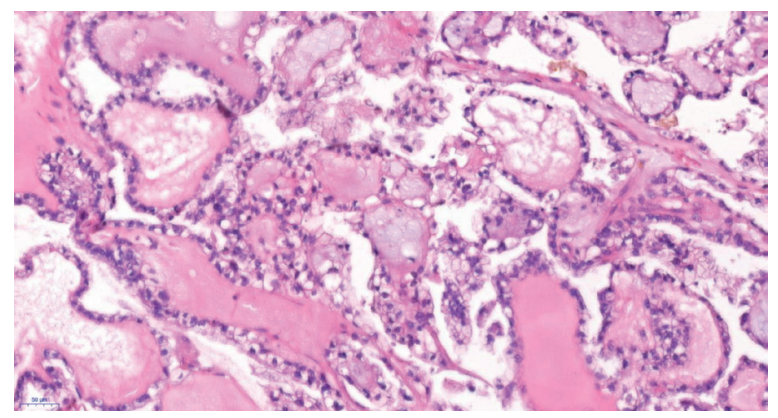

Figure 4. Microphotograph presenting H\&E stained specimens of clear cell ovarian cancer, primary objective magnification $20 \times$ of ovarian carcinomas, especially mucinous tumors [39]. Similar frequency of KRAS mutations was reported for ovarian endometrioid carcinomas (7\%), which supports the hypothesis about a common origin of these two tumor types [39]. According to Zannoni et al., KRAS mutations found in CCCs are located in codon 12, exon 2, but not in codon 13, exon 2 [40].

\section{Determination of BRAF, KRAS and NRAS mutation status}

Molecular analysis of cancer-causing mutations and development of targeted biological therapies constitute a milestone in diagnosis and therapy of ovarian malignancies. Until a few years ago, classification of cancers was based primarily on the type of tissue they arose, histopathological and clinical characteristics. However, more recently, oncological diagnosis has been also expanded by including molecular features of cancer cells. Usually, molecular tests become a component of routine patient care if detection of a specific alteration may have impact on diagnosis and/or prognosis, or whenever a targeted biological therapy is available.

Point mutations in cancer cells can be detected with many various methods. KRAS, NRAS and BRAF mutational status can be determined by direct sequencing, which can detect all potential variations, including base substitutions, insertions and deletions. However, direct sequencing has some limitations when applied to clinical samples. First of all, it is not sensitive enough (10-30\%) to detect specific point mutations. Analytic sensitivity of this method can be improved by pyrosequencing, high-resolution melting analysis, real-time or allele-specific PCR assays. Next-generation sequencing methods provide high analytic sensitivity, offer broad reportable range of mutation spectrum, and have adequate capacity for quantitative measurement of mutant allele frequencies and simultaneous detection of concomitant mutations [41, 42].

Application of direct sequencing as a routine method for cytological diagnosis used in a hospital setting requires expensive equipment and implementation of complicated procedures. Another factor limiting application of this method in everyday clinical practice are long analytical times. This stimulated search for a simple, rapid, specific and sensitive methodology to detect point mutations. Recently, some new molecular assays for the detection of BRAF, KRAS and NRAS mutations have become available (Fig. 5). These are fully-automated molecular diagnostic systems for quantitative allele-specific RT-PCR-based analyses. Unlike currently available technologies, Idylla ${ }^{\mathrm{TM}}$ Mutation Test does not require manual preprocessing of the sample (deparaffinization, digestion of FFPE tissue or DNA extraction), since all these procedures are integrated within a single-use cartridge. Instead, whole FFPE tissue sections or macro-dissected FFPE specimens are inserted into the cartridge and 




Figure 5. Microphotographs showing immunoexpression of BRAF V600E (VE1) protein various histological types of ovarian tumors

processed by the Idylla ${ }^{\mathrm{TM}}$ system; also further stages, i.e. real-time PCR-based mutation detection and result reporting, are fully-integrated and automated [43]. Without the need for highly-skilled staff, mutational tests approved by the European Community for in vitro diagnostic use (labeled as (E-IVD) can genotype KRAS, BRAF and NRAS with a high detection limit, and therefore, can be used as a standardized method, even at diagnostic centers without a developed molecular infrastructure [44]. Using this instrument, even pathologists from less experienced laboratories can easily integrate morphological findings with molecular data being crucial for further diagnostic and therapeutic decisions.

\section{CONCLUSIONS}

In recent years, a plethora of compounds targeting components of RAS-RAF-MEK pathway entered clinical trials, and some of them have already demonstrated a promising clinical activity. Beneficial effects of targeted therapies may be associated with inhibition of downstream signaling pathways, primarily RAS-RAF-MAPK and PI3K-PTEN-AKT. Introduction of personalized therapies has revolutionized standards of care in selected cases; detection of a critical somatic mutation in cancer cells improves diagnostic and prognostic accuracy, but first of all, may guide the selection of a highly-effective targeted therapy, producing not only health but also economic benefits.

\section{Acknowledgements}

We would like to acknowledge dr hab. n. med. Dariusz Grzanka, dr n. med. Magdalena Bodnar and mgr Paulina Antosik from Department of Clinical Pathomorphology, Collegium
Medicum in Bydgoszcz, Nicolaus Copernicus University in Torun, Bydgoszcz, Poland for microphotographs of tumor tissues.

\section{REFERENCES}

1. Gąsiorowska E, Michalak M, WarchołW, et al. Clinical application of HE4 and CA125 in ovarian cancer type I and type II detection and differential diagnosis. Ginekol Pol. 2015; 86(2): 88-93, indexed in Pubmed: 25807831.

2. Shih IM, Kurman RJ. Ovarian tumorigenesis: a proposed model based on morphological and molecular genetic analysis. Am J Pathol. 2004; 164(5): 1511-1518, indexed in Pubmed: 15111296.

3. Shaw PA, McLaughlin JR, Zweemer RP, et al. Histopathologic features of genetically determined ovarian cancer. Int J Gynecol Pathol. 2002; 21(4): 407-411, doi: 10.1097/00004347-200210000-00011, indexed in Pubmed: 12352190.

4. Skead G, Govender D. Gene of the month: MET. J Clin Pathol. 2015; 68(6): 405-409, doi: 10.1136/jclinpath-2015-203050, indexed in Pubmed: 25987653.

5. Huang J, Zhang L, Greshock J, et al. Frequent genetic abnormalities of the PI3K/AKT pathway in primary ovarian cancer predict patient outcome. Genes Chromosomes Cancer. 2011; 50(8): 606-618, doi: 10.1002/gcc.20883, indexed in Pubmed: 21563232.

6. Olson JM, Hallahan AR. p38 MAP kinase: a convergence point in cancer therapy. Trends Mol Med. 2004; 10(3): 125-129, doi: 10.1016/j.molmed.2004.01.007, indexed in Pubmed: 15102355.

7. Hsu CY, Bristow R, Cha MS, et al. Characterization of active mitogen-activated protein kinase in ovarian serous carcinomas. Clin Cancer Res. 2004; 10(19): 6432-6436, doi: 10.1158/1078-0432.CCR-04-0893, indexed in Pubmed: 15475429.

8. Grabowski JP, Harter P, Heitz F, et al. Operability and chemotherapy responsiveness in advanced low-grade serous ovarian cancer. An analysis of the AGO Study Group metadatabase. Gynecol Oncol. 2016; 140(3): 457-462, doi: 10.1016/j.ygyno.2016.01.022, indexed in Pubmed: 26807488.

9. Ramalingam P. Morphologic, Immunophenotypic, and Molecular Features of Epithelial Ovarian Cancer. Oncology (Williston Park). 2016; 30(2): 166-176, indexed in Pubmed: 26892153.

10. Malpica A, Deavers MT. Ovarian low-grade serous carcinoma involving the cervix mimicking a cervical primary. Int J Gynecol Pathol. 2011; 30(6): 613-619, doi: 10.1097/PGP.0b013e318217137e, indexed in Pubmed: 21979600

11. Phillips V, Kelly $P$, McCluggage WG. Increased $\mathrm{p} 16$ expression in high-grade serous and undifferentiated carcinoma compared with other morphologic types of ovarian carcinoma. Int J Gynecol Pathol. 
2009; 28(2): 179-186, doi: 10.1097/PGP.0b013e318182c2d2, indexed in Pubmed: 19188815.

12. Singer $G$, Stöhr R, Cope $L$, et al. Patterns of $p 53$ mutations separate ovarian serous borderline tumors and low- and high-grade carcinomas and provide support for a new model of ovarian carcinogenesis: a mutational analysis with immunohistochemical correlation. Am J Surg Pathol. 2005; 29(2): 218-224, doi: 10.1097/01.pas.0000146025.91953.8d, indexed in Pubmed: 15644779.

13. Grisham RN, lyer G, Garg K, et al. BRAF mutation is associated with early stage disease and improved outcome in patients with low-grade serous ovarian cancer. Cancer. 2013; 119(3): 548-554, doi: 10.1002/cncr.27782, indexed in Pubmed: 22930283.

14. Zeppernick F, Ardighieri L, Hannibal CG, et al. BRAF mutation is associated with a specific cell type with features suggestive of senescence in ovarian serous borderline (atypical proliferative) tumors. Am J Surg Pathol. 2014; 38(12): 1603-1611, doi: 10.1097/PAS.0000000000000313, indexed in Pubmed: 25188864.

15. Singer G, Oldt R, Cohen Y, et al. Mutations in BRAF and KRAS characterize the development of low-grade ovarian serous carcinoma. J Natl Cancer Inst. 2003; 95(6): 484-486, doi: 10.1093/jnci/95.6.484, indexed in Pubmed: 12644542.

16. Houghton O, Connolly LE, McCluggage WG. Morules in endometrioid proliferations of the uterus and ovary consistently express the intestinal transcription factor CDX2. Histopathology. 2008; 53(2): 156-165, doi: 10.1111/j.1365-2559.2008.03083.x, indexed in Pubmed: 18752499.

17. Yemelyanova A, Ji H, Shih IM, et al. Utility of $\mathrm{p} 16$ expression for distinction of uterine serous carcinomas from endometrial endometrioid and endocervical adenocarcinomas: immunohistochemical analysis of 201 cases. Am J Surg Pathol. 2009; 33(10): 1504-1514, doi: 10.1097/PAS.0b013e3181ac35f5, indexed in Pubmed: 19623034.

18. Soliman PT, Broaddus RR, Schmeler KM, et al. Women with synchronous primary cancers of the endometrium and ovary: do they have Lynch syndrome? J Clin Oncol. 2005; 23(36): 9344-9350, doi: 10.1200/JCO.2005.03.5915, indexed in Pubmed: 16361634.

19. Catasús L, Bussaglia E, Rodrguez I, et al. Molecular genetic alterations in endometrioid carcinomas of the ovary: similar frequency of beta-catenin abnormalities but lower rate of microsatellite instability and PTEN alterations than in uterine endometrioid carcinomas. Hum Pathol. 2004; 35(11): 1360-1368, doi: 10.1016/j.humpath.2004.07.019, indexed in Pubmed: 15668893.

20. Silva EG, Deavers MT, Malpica A. Patterns of low-grade serous carcinoma with emphasis on the nonepithelial-lined spaces pattern of invasion and the disorganized orphan papillae. Int J Gynecol Pathol. 2010; 29(6): 507-512, doi: 10.1097/PGP.0b013e3181e31f74, indexed in Pubmed: 20881864.

21. Taraif SH, Deavers MT, Malpica A, et al. The significance of neuroendocrine expression in undifferentiated carcinoma of the endometrium. Int J Gynecol Pathol. 2009; 28(2): 142-147, doi: 10.1097/PGP.0b013e3181895539, indexed in Pubmed: 19188820.

22. Kurman RJ, Shih IM. Molecular pathogenesis and extraovarian origin of epithelial ovarian cancer--shifting the paradigm. Hum Pathol. 2011; 42(7): 918-931, doi: 10.1016/j.humpath.2011.03.003, indexed in Pubmed: 21683865

23. Ryland G, Hunter S, Doyle M, et al. Mutational landscape of mucinous ovarian carcinoma and its neoplastic precursors. Genome Med. 2015; 7(1), doi: 10.1186/s13073-015-0210-y.

24. Seidman JD, Kurman RJ, Ronnett BM. Primary and metastatic mucinous adenocarcinomas in the ovaries: incidence in routine practice with a new approach to improve intraoperative diagnosis. Am J Surg Pathol. 2003; 27(7): 985-993, doi: 10.1097/00000478-200307000-00014, indexed in Pubmed: 12826891

25. Alexandre J, Ray-Coquard I, Selle F, et al. GINECO. Mucinous advanced epithelial ovarian carcinoma: clinical presentation and sensitivity to platinum-paclitaxel-based chemotherapy, the GINECO experience. Ann Oncol. 2010; 21(12): 2377-2381, doi: 10.1093/annonc/mdq257, indexed in Pubmed: 20494964.

26. Anglesio MS, Kommoss S, Tolcher MC, et al. Molecular characterization of mucinous ovarian tumours supports a stratified treatment approach with HER2 targeting in 19\% of carcinomas. J Pathol. 2013; 229(1): 111-120, doi: 10.1002/path.4088, indexed in Pubmed: 22899400.
27. Grisham RN, lyer G, Garg K, et al. BRAF mutation is associated with early stage disease and improved outcome in patients with low-grade serous ovarian cancer. Cancer. 2013; 119(3): 548-554, doi: 10.1002/cncr.27782, indexed in Pubmed: 22930283.

28. Gemignani ML, Schlaerth AC, Bogomolniy F, et al. Role of KRAS and BRAF gene mutations in mucinous ovarian carcinoma. Gynecol Oncol. 2003; 90(2): 378-381, doi: 10.1016/s0090-8258(03)00264-6, indexed in Pubmed: 12893203.

29. Rechsteiner M, Zimmermann AK, Wild PJ, et al. TP53 mutations are common in all subtypes of epithelial ovarian cancer and occur concomitantly with KRAS mutations in the mucinous type. Exp Mol Pathol. 2013; 95(2): 235-241, doi: 10.1016/j.yexmp.2013.08.004, indexed in Pubmed: 23965232.

30. Chan JK, Teoh D, Hu JM, et al. Do clear cell ovarian carcinomas have poorer prognosis compared to other epithelial cell types? A study of 1411 clear cell ovarian cancers. Gynecol Oncol. 2008; 109(3): 370-376, doi: 10.1016/j.ygyno.2008.02.006, indexed in Pubmed: 18395777.

31. Sugiyama T, Kamura T, Kigawa J, et al. Clinical characteristics of clear cell carcinoma of the ovary: a distinct histologic type with poor prognosis and resistance to platinum-based chemotherapy. Cancer. 2000; 88(11): 2584-2589, indexed in Pubmed: 10861437.

32. Wu RC, Ayhan A, Maeda D, et al. Frequent somatic mutations of the telomerase reverse transcriptase promoter in ovarian clear cell carcinoma but not in other major types of gynaecological malignancy. J Pathol. 2014; 232(4): 473-481, doi: 10.1002/path.4315, indexed in Pubmed: 24338723.

33. Köbel M, Reuss $A$, du Bois $A$, et al. The biological and clinical value of p53 expression in pelvic high-grade serous carcinomas. J Pathol. 2010; 222(2): 191-198, doi: 10.1002/path.2744, indexed in Pubmed: 20629008.

34. Kuo KT, Mao TL, Chen Xu, et al. Frequent activating mutations of PIK3CA in ovarian clear cell carcinoma. Am J Pathol. 2009; 174(5): 1597-1601, doi: 10.2353/ajpath.2009.081000, indexed in Pubmed: 19349352.

35. Willner J, Wurz K, Allison KH, et al. Alternate molecular genetic pathways in ovarian carcinomas of common histological types. Hum Pathol. 2007; 38(4): 607-613, doi: 10.1016/j.humpath.2006.10.007, indexed in Pubmed: 17258789 .

36. Jones $\mathrm{S}$, Wang $\mathrm{TL}$, Shih $\mathrm{IM}$, et al. Frequent mutations of chromatin remodeling gene ARID1A in ovarian clear cell carcinoma. Science. 2010; 330(6001): 228-231, doi: 10.1126/science.1196333, indexed in Pubmed: 20826764

37. Auner V, Kriegshäuser $G$, Tong $D$, et al. KRAS mutation analysis in ovarian samples using a high sensitivity biochip assay. BMC Cancer. 2009; 9: 111, doi: 10.1186/1471-2407-9-111, indexed in Pubmed: 19358724.

38. Rechsteiner M, Zimmermann AK, Wild PJ, et al. TP53 mutations are common in all subtypes of epithelial ovarian cancer and occur concomitantly with KRAS mutations in the mucinous type. Exp Mol Pathol. 2013; 95(2): 235-241, doi: 10.1016/j.yexmp.2013.08.004, indexed in Pubmed: 23965232.

39. Despierre E, Yesilyurt BT, Lambrechts S, et al. EORTC GCG and EORTC GCG Translational Research Group. Epithelial ovarian cancer: rationale for changing the one-fits-all standard treatment regimen to subtype-specific treatment. Int J Gynecol Cancer. 2014; 24(3): 468-477, doi: 10.1097/IGC.0000000000000089, indexed in Pubmed: 24557434.

40. Zannoni GF, Improta G, Chiarello G, et al. Mutational status of KRAS, NRAS, and BRAF in primary clear cell ovarian carcinoma. Virchows Arch. 2014; 465(2): 193-198, doi: 10.1007/s00428-014-1599-1, indexed in Pubmed: 24889043.

41. Anderson SM. Laboratory methods for KRAS mutation analysis. Expert Rev Mol Diagn. 2011; 11(6): 635-642, doi: 10.1586/erm.11.42, indexed in Pubmed: 21745016.

42. Haley L, Tseng LH, Zheng G, et al. Performance characteristics of next-generation sequencing in clinical mutation detection of colorectal cancers. Mod Pathol. 2015; 28(10): 1390-1399, doi: 10.1038/modpathol.2015.86, indexed in Pubmed: 26226847

43. Janku F, Huang $\mathrm{HJ}$, Claes $\mathrm{B}$, et al. BRAF mutation testing with a rapid, fully integrated molecular diagnostics system. Oncotarget. 2015; 6(29): 26886-26894, doi: 10.18632/oncotarget.4723, indexed in Pubmed: 26330075.

44. Sadlecki $P$, Walentowicz $P$, Bodnar $M$, et al. Determination of BRAFV600E (VE1) protein expression and BRAF gene mutation status in codon 600 in borderline and low-grade ovarian cancers. Tumour Biol. 2017; 39(5): 1010428317706230, doi: 10.1177/1010428317706230, indexed in Pubmed: 28488545. 